\title{
Statistical analysis of compressive low rank tomography with random measurements
}

\author{
Anirudh Acharya, Mădălin Guţă \\ School of Mathematical Sciences, University of Nottingham, University Park, NG7 2RD \\ Nottingham, United Kingdom
}

\begin{abstract}
.
We consider the statistical problem of 'compressive' estimation of low rank states ( $r \ll$ $d$ ) with random basis measurements, where $r, d$ are the rank and dimension of the state respectively. We investigate whether for a fixed sample size $N$, the estimation error associated with a 'compressive' measurement setup is 'close' to that of the setting where a large number of bases are measured. We generalise and extend previous results, and show that the mean square error (MSE) associated with the Frobenius norm attains the optimal rate $r d / N$ with only $O(r \log d)$ random basis measurements for all states. An important tool in the analysis is the concentration of the Fisher Information Matrix (FIM). We demonstrate that although a concentration of the MSE follows from a concentration of the FIM for most states, the FIM fails to concentrate for states with eigenvalues close to zero.

We analyse this phenomenon in the case of a single qubit and demonstrate a concentration of the MSE about its optimal despite a lack of concentration of the FIM for states close to the boundary of the Bloch sphere. We also consider the estimation error in terms of a different metric-the quantum infidelity. We show that a concentration in the mean infidelity (MINF) does not exist uniformly over all states, highlighting the importance of loss function choice. Specifically, we show that for states that are nearly pure, the MINF scales as $1 / \sqrt{N}$ but the constant converges to zero as the number of settings is increased. This demonstrates a lack of 'compressive' recovery for nearly pure states in this metric.
\end{abstract}

\section{Introduction}

Quantum information tasks require the experimental preparation, control and transformation of individual quantum systems [1,2]. An important element in such tasks in often the use of quantum state tomography (QST) to validate the results $[3,4]$. The aim of QST it to statistically reconstruct the density matrix associated with the unknown state from outcomes of repeated measurements performed on identical copies of the state. There are several estimation methods used for state reconstruction, and here we only mention a few relevant examples such as maximum likelihood $[5,6,7,8,9]$, estimation with incomplete measurements [13, 14, 15], linear inversion and Bayesian inference [10, 11, 12].

However full state tomography often becomes challenging due to the exponential increase in the dimension of the model. There is significant interest in addressing this challenge and as a result, extensive work has been done in developing tomography methods for certain lower dimensional families of physically relevant states. Pertinent examples include the estimation of low rank states in the context of compressed sensing (CS) [18, 19, 20, 21, 22], model selection [23], and spectral thresholding [24,25]. Similarly, the estimation of matrix product 
states [26] is particularly relevant for many-body systems, but also for estimating dynamical parameters of open systems [27, 28].

Based on the compressed sensing idea, several recent papers [29, 30,31] consider the problem of estimating low rank states from random measurements. Inspired by the PhaseLift problem, the papers [29, 32, 33] consider the case of estimating low rank states from expectations of rank-one projections sampled randomly from a Gaussian distribution, or a projective t-design, and demonstrate stable compressive recovery with estimation errors of the order of the number of unknown parameters. Compressive quantum process tomography has been considered in this context for unitary 2-designs [34]. In [35] the analysis is extended to the physically relevant case of random orthonormal basis measurements, and it is shown that a rank- $r$ state can be identified with a large probability for only $O\left(r \log ^{3} d\right)$ such random measurements. Related to this question of low-rank state estimation, work in [36] conjectures that only a few random bases correspond to strictly complete POVMs for low rank states, implying that states of a given low rank can be compressively recovered by measuring a small number of random bases, independent of dimension.

In this paper we build on the work in [31], and consider the statistical problem of estimating low-rank states in the set up of random bases measurements. Instead of choosing a particular estimator, the idea is to investigate the statistical efficiency of an arbitrary optimal estimator, and find whether rank-r states can be estimated from only a few random bases measurements. For this, we consider the behaviour of the Mean Square Error (MSE) with respect to the Frobenius distance between the true state and the estimator $\|\hat{\rho}-\rho\|_{2}^{2}$ in the limit of large number of measurement samples. According to asymptotic theory [37], in the regime of large number of repetitions the MSE of efficient estimators (e.g. maximum likelihood) $\hat{\rho}$ takes the following expression

$$
\mathbb{E}\|\hat{\rho}-\rho\|_{2}^{2}=\frac{1}{N} \operatorname{Tr}\left(I(\rho \mid \mathcal{S})^{-1} G_{F}\right)+o\left(N^{-1}\right) .
$$

Above, $I(\rho \mid \mathcal{S})$ is the classical Fisher information associated with the chosen measurement design $\mathcal{S}$ and a local parametrisation of rank- $r$ states, $N$ is the total number of measured systems, and $G_{F}$ is the positive weight matrix associated with the quadratic approximation of the Frobenius distance in the local parameters.

The asymptotic MSE (1) has been shown to remain robust even with only a few random basis measurements making up the design $\mathcal{S}$ [31]. This robustness is explained using an argument based on a concentration inequality [38] for the Fisher information matrix. It is shown in [31] that certain 'least sparse' states of rank-r can be estimated by using only $O(r \log d)$ settings with only a small increase in the MSE, relative to the setup in which a large number of settings is probed. In this paper the argument using the concentration of the Fisher information is extended to hold for all rank-r states (Theorem 1), incorporating the results in [31]. However, we discuss drawbacks of using a concentration in the Fisher information to derive a corresponding concentration in the MSE. Specifically, for rank-r states that are close to pure with small eigenvalues, we show that such a concentration of the Fisher information does not hold. This difficulty is overcome by proving an upper bound on the MSE that holds for all states independently of their spectrum. We show that $\operatorname{Tr}\left(I(\rho \mid \mathcal{S})^{-1} G_{F}\right)$ is bounded from above by roughly the number of unknown parameters given that $O(r \log d)$ random bases constitute the measurement design $\mathcal{S}$. As an illustrative example, we consider a single qubit state and analyse the failure of the Fisher concentration for states that are close to pure. We argue that despite a lack of concentration in the Fisher information for such states, the MSE demonstrates the necessary concentration. 
The lack of concentration of the Fisher information occurs as the elements of the matrix corresponding to the small eigenvalues of the state diverge. However for states that are known to be pure and have $d-1$ eigenvalues exactly zero, the Fisher information matrix contains entries that correspond only to the 'rotation' parameters. Therefore it concentrates following Theorem 1, and [31] (Theorem 4.1) given that $O(\log d)$ random basis are measured. Related work in a different context [45] shows that (local) informationally complete measurements for pure states require the number of outcomes to scale linearly with the dimension $d$, specifically $2 d-1$. In our measurement design, the total number of outcomes is $O(d \log d)$ for pure states. For the single qubit case, we also investigate the problem of 'compressive' state estimation using the quantum infidelity $1-F(\hat{\rho}, \rho)=1-\operatorname{Tr}(\sqrt{\sqrt{\rho} \hat{\rho} \sqrt{\rho}})^{2}$ as the error metric. For this we consider the asymptotic mean infidelity (MINF),

$$
\mathbb{E}[1-F(\hat{\rho}, \rho)]=\frac{1}{N} \operatorname{Tr}\left(I(\rho \mid \mathcal{S})^{-1} G_{I N F}\right)
$$

with the Fisher information as defined in (1), and $G_{I N F}$ being the weight matrix corresponding to the quadratic approximation of the infidelity. Unlike the Frobenius distance, the quantum infidelity is very sensitive to the misestimation of small eigenvalues. In particular, for states that are close to pure with small eigenvalues, the local expansion of the infidelity in the asymptotic regime is linear in the estimation error of these eigenvalues [39]. This means that the MINF is no longer given by quadratic expression (2) for such states. We show that for states with eigenvalues well away from zero, a concentration in the MINF given by (2) can be demonstrated using a concentration of the Fisher information matrix. While for nearly pure states and random measurements both the Fisher information and the MINF demonstrate a lack of concentration. For such states the MINF scales as $O(1 / \sqrt{N})$, and additionally there is no finite number of settings such that the state can be estimated 'compressively'.

\section{Quantum tomography with random basis measurements}

In this paper we consider the problem of estimating an unknown quantum state represented by a $d \times d$ density matrix $\rho$ (complex, positive trace-one matrix), where $d$ is the dimension of the associated Hilbert space $\mathcal{H}^{d}$. The unknown state is reconstructed from the outcomes of projective measurements on identical copies of the state. The measurement settings are chosen by randomly drawing an orthonormal basis (ONB) from the uniform measure, or equivalently by rotating a fixed (standard) ONB with a random unitary $U$ drawn from the Haar measure over the unitaries on $\mathcal{H}^{d}$. We denote measurement settings by $\mathbf{s}$ and the corresponding ONBs by $\left\{\left|e_{\mathbf{s}}^{\mathbf{o}}\right\rangle\right\}$ where $\mathbf{o} \in\{1, \ldots, d\}$ is the label of a measurement outcome. Its probability is $p_{\rho}(\mathbf{o} \mid \mathbf{s}):=\operatorname{Tr}\left(\rho P_{\mathbf{o}}^{\mathbf{s}}\right)$, where $P_{\mathbf{o}}^{\mathbf{s}}=\left|e_{\mathbf{s}}^{\mathbf{o}}\right\rangle\left\langle e_{\mathbf{s}}^{\mathbf{o}}\right|$ is the one-dimensional projection corresponding to the outcome $\mathbf{o}$, in the measurement setting $\mathbf{s}$. Because of the cyclicity of the trace, this measurement design is mathematically equivalent to fixing a particular measurement basis and rotating the state $\rho$ with a known random unitary corresponding to the measurement design.

This design is motivated by the multiple ion tomography (MIT) set up of ion-trap experiments [3] considered in our previous work [31]. The aim of MIT is to determine the unknown density matrix $\rho \in \mathcal{H}^{d}$ of the joint state of a system of $n$ ions, where $d=2^{n}$ is the dimension of the associated Hilbert space. A random measurement setting $\mathbf{s}$ in the MIT setup can be thought of as a rotation of the fixed $\sigma_{z}^{\otimes n}$ basis by a random unitary drawn from the Haar measure over the whole Hilbert space $\mathcal{H}^{2^{n^{2}}}$.

The measurement procedure and statistical model are summarised below, following the notation of our previous work [31]. For each given setting, the measurement is repeated 
on $m$ copies of the state. This is repeated for all $k$ settings, and the total number of copies of the state utilised as a resource is $N=m \times k$. This procedure results in information about the number of times a particular outcome was observed for a given setting $N(\mathbf{o} \mid \mathbf{s})$. This information can be thought of as a $d \times k$ dataset of counts whose columns are independent. In this paper we investigate the statistical efficiency of estimating low rank states from such measurement outcomes. We work in the asymptotic regime in which the number of repetitions $m$ in each setting is large, and characterise the estimation errors in terms of the classical Fisher information matrix as explained below.

We first introduce a parametrisation of the state $\rho$, and assume that the state has rank $r \leq d$, and therefore belongs to the space of rank $r$ states $\mathbb{S}_{r} \subset M\left(\mathbb{C}^{d}\right)$. In the asymptotic scenario the estimation error is characterised by a local statistical model. Therefore, we consider a local parametrisation $\boldsymbol{\theta} \rightarrow \rho_{\boldsymbol{\theta}}$ of the state $\rho$ in the space $\mathbb{S}_{r}$. In this space, any rank-r state $\rho^{\prime}$ in the local neighbourhood of $\rho$ can be obtained by a perturbation of the eigenvalues of $\rho$, along with a small rotation of the eigenbasis. In the first order this transformation leaves the $(d-r) \times(d-r)$ lower-right corner unchanged, so that in the eigenbasis of the state $\rho$ we have

$$
\rho^{\prime}=\left(\begin{array}{cc}
\operatorname{Diag}\left(\lambda_{1}, \ldots, \lambda_{r}\right) & 0 \\
0 & 0
\end{array}\right)+\left(\begin{array}{cc}
\Delta_{\text {diag }} & \Delta_{o f f} \\
\Delta_{o f f}^{\dagger} & O\left(\|\Delta\|^{2}\right)
\end{array}\right) .
$$

We therefore choose to parametrise such a state $\rho^{\prime}=\rho_{\boldsymbol{\theta}}^{\prime}$ with

$$
\begin{aligned}
\boldsymbol{\theta} & :=\left(\boldsymbol{\theta}^{(d)} ; \boldsymbol{\theta}^{(r)} ; \boldsymbol{\theta}^{(i)}\right) \\
& =\left(\rho_{2,2}^{\prime}, \ldots, \rho_{r, r}^{\prime} ; \operatorname{Re} \rho_{1,2}^{\prime}, \ldots, \operatorname{Re} \rho_{r, d}^{\prime} ; \operatorname{Im} \rho_{1,2}^{\prime}, \ldots, \operatorname{Im} \rho_{r, d}^{\prime}\right) \in \mathbb{R}^{2 r d-r^{2}-1}
\end{aligned}
$$

where, the first diagonal matrix element does not appear in the parametrisation as it is fixed by the trace normalisation of density matrices. We can now describe the statistical model in this parametrisation, and define the classical Fisher information matrix associated with a given setting $\mathbf{s}$ as

$$
I(\rho \mid \mathbf{s})_{a, b}:=\sum_{\mathbf{o}: p(\mathbf{o} \mid \mathbf{s})>0} \frac{1}{p_{\rho}(\mathbf{o} \mid \mathbf{s})} \frac{\partial p_{\rho}(\mathbf{o} \mid \mathbf{s})}{\partial \theta_{a}} \cdot \frac{\partial p_{\rho}(\mathbf{o} \mid \mathbf{s})}{\partial \theta_{b}} .
$$

Where $\theta_{a, b}$ are labelled elements of the parameter vector $\boldsymbol{\theta}$. Following the measurement procedure described above, we define the set of $k$ measurement settings as $\mathcal{S}$. The Fisher information matrix associated with a single measurement from each setting $\mathbf{s} \in \mathcal{S}$ is given by the sum of the individual Fisher matrices above. The average Fisher information for the measurement design $\mathcal{S}$ is denoted as $I(\rho \mid \mathcal{S})=\frac{1}{k} \sum_{\mathbf{s} \in \mathcal{S}} I(\rho \mid \mathbf{s})$. The individual matrices are computed using definition (5) together with parametrisation (4).

The measurement in each setting is repeated $m$ times on identical copies of the state, and the outcomes are i.i.d. When this number $m$ is sufficiently large, efficient estimators of $\boldsymbol{\theta}$ (and hence of $\rho$ ) from these outcomes have an asymptotically Gaussian distribution [37]

$$
\sqrt{m}(\hat{\boldsymbol{\theta}}-\boldsymbol{\theta}) \approx \mathcal{N}\left(0, I(\rho \mid \mathcal{S})^{-1}\right)
$$

where the covariance matrix $I(\rho \mid \mathcal{S})^{-1}$ is the Fisher information associated with a single measurement sample of the set $\mathcal{S}$. In the following section, this asymptotic behaviour of the estimate $\hat{\boldsymbol{\theta}}$ is combined with local expansions of the Frobenius distance in order to characterise the MSE in terms of the classical Fisher information matrix. In section 4.1 the asymptotic mean infidelity (MINF) is investigated using similar techniques. 


\section{Bounds for the MSE}

We now consider the Frobenius distance, and characterise the efficiency of any efficient estimator (such as maximum likelihood) in its terms. This distance has a locally quadratic expansion around the state $\rho$, given by

$$
\left\|\rho_{\boldsymbol{\theta}}-\rho_{\boldsymbol{\theta}+\boldsymbol{\delta} \boldsymbol{\theta}}\right\|_{2}^{2}=(\boldsymbol{\delta} \boldsymbol{\theta})^{T} G_{F}(\boldsymbol{\delta} \boldsymbol{\theta})+o\left(\|\boldsymbol{\delta} \boldsymbol{\theta}\|^{2}\right)
$$

where $G_{F}$ is a constant weight matrix that reproduces the Frobenius norm. The explicit form of this weight matrix can be found in the appendix. From this and the asymptotic behaviour of efficient estimators, we see that for (reasonably) large $m$, the mean square error scales as

$$
\operatorname{MSE}:=\mathbb{E}\left(\|\hat{\rho}-\rho\|_{2}^{2}\right) \approx \frac{1}{N} \operatorname{Tr}\left(I(\rho \mid \mathcal{S})^{-1} G_{F}\right) .
$$

The expression of the right side of the above equation is a measure of the sensitivity of the chosen set of settings $\mathcal{S}$ at $\rho$ [31]. We therefore consider the behaviour of the MSE in terms of this trace expression, and study the effect of the measurement design $\mathcal{S}$ and the number of settings $k$ on the error. We first present a preliminary concentration bound for this quantity $\operatorname{Tr}\left(I(\rho \mid \mathcal{S})^{-1} G_{F}\right)$, which extends the results in [31].

The bound determines the number of settings $k$ required for the MSE $\operatorname{Tr}\left(I(\rho \mid \mathcal{S})^{-1} G_{F}\right)$ to be concentrated close its optimal value. This result is derived from a concentration of the Fisher information matrix around the mean Fisher information, where the main ingredient is a matrix Chernoff bound for sums of bounded random Hermitian matrices. Since the settings in $\mathcal{S}$ are independent, the Fisher information matrices $I(\rho \mid \mathbf{s})$ are independent and this bound is applicable. The Chernoff bound determines how quickly the average information per setting $\frac{1}{k} \sum_{k \in \mathcal{S}} I(\rho \mid \mathbf{s})$ approaches the mean information $\bar{I}$ over all random settings. In terms of the MSE, this translates to determining the number of settings $k$ required for the MSE $\operatorname{Tr}\left(I(\rho \mid \mathcal{S})^{-1} G_{F}\right)$ to be concentrated close the optimal value of $\operatorname{Tr}\left(\bar{I}(\rho)^{-1} G_{F}\right)$. We consider states with arbitrary spectrums $\rho:=\operatorname{Diag}\left(\lambda_{1}, \ldots, \lambda_{r}, \ldots, 0\right)$, diagonal with respect to its eigenbasis. Due to the unitary symmetry of the random settings design, the eigenbasis can be chosen to be the standard basis.

Theorem 1. Let $\mathcal{S}=\left\{\mathbf{s}_{1}, \ldots, \mathbf{s}_{k}\right\}$ be a design with randomly, uniformly distributed measurement bases. Let $I_{\mathcal{S}}:=I(\rho \mid \mathcal{S})$ be the associated Fisher information, and let $\bar{I}$ be the mean Fisher information over all possible bases, both calculated at the true state $\rho$. For a sufficiently small $\epsilon \geq 0$, the following inequality holds

$$
(1-\epsilon) \operatorname{Tr}\left[\bar{I}^{-1} G_{F}\right] \leq \operatorname{Tr}\left[I_{\mathcal{S}}^{-1} G_{F}\right] \leq(1+\epsilon) \operatorname{Tr}\left[\bar{I}^{-1} G_{F}\right]
$$

with probability $1-\delta$, provided that the number of measurements performed is $k=$ $\frac{C_{1}}{\lambda_{\min }(\rho)} \frac{(r+1)}{r} \log \left(\frac{2 D}{\delta}\right)$, with $D=2 r d-r^{2}-1$ the dimension of the space of rank-r states, and $C_{1}=4\left(\log 2 / \epsilon^{2}\right)$.

The proof of this theorem and further details can be found in the appendix. As mentioned earlier, the main ingredient is a matrix Chernoff bound [38], which is used to bound the deviation of $G_{F}^{-/ 2} I(\rho \mid \mathcal{S}) G_{F}^{-1 / 2}$ from the mean $G_{F}^{-/ 2} \bar{I}(\rho) G_{F}^{-1 / 2}$. The number of uniformly random settings $k$ required in the theorem above depends on the following ratio

$$
\frac{\mu_{\max }}{\mu_{\min }}:=\frac{\max _{\mathbf{s}} \lambda_{\max }\left(G_{F}^{-1 / 2} I(\rho \mid \mathbf{s}) G_{F}^{-1 / 2}\right)}{\lambda_{\min }\left(G_{F}^{-1 / 2} \bar{I}(\rho) G_{F}^{-1 / 2}\right)}
$$


between the largest maximum eigenvalue of $G_{F}^{-1 / 2} I(\rho \mid \mathbf{s}) G_{F}^{-1 / 2}$ over all possible measurements and the minimum eigenvalue of $G_{F}^{-1 / 2} \bar{I}(\rho) G_{F}^{-1 / 2}$. Details of the explicit values of this ratio is left to the appendix. The numerator $\mu_{\max }$ is upper bounded by using the inequality between the quantum and classical Fisher informations [40], as $\mu_{\max } \leq 2 / \lambda_{\min }(\rho)$ for $r>1$ and $\mu_{\max } \leq 2$ for $r=1$. While the minimum eigenvalue of $G_{F}^{-1 / 2} \bar{I}(\rho) G_{F}^{-1 / 2}$ is lower bounded using the following lemma.

Lemma 1. For any rank-r state $\rho$ with an arbitrary spectrum, and the rank-r state $\rho_{0}$ which has equal non-zero eigenvalues $1 / r$ and the same eigenvectors as $\rho$, the following inequality holds between their average Fisher information matrices, evaluated over all possible random measurement settings.

$$
\bar{I}\left(\rho_{0}\right) \leq \bar{I}(\rho)
$$

The proof is left to the appendix. The matrix $\bar{I}\left(\rho_{0}\right)$ for the equal eigenvalue state has been computed explicitly by using analytic expressions for moments of random unitaries [41], which gives $\mu_{\min } \geq \frac{r}{r+1}$ for $r>1$, and $\mu_{\min } \geq 1$ for pure states. Together these give $\frac{\mu_{\max }}{\mu_{\min }} \leq 2 \frac{(r+1)}{r} \frac{1}{\lambda_{\min }(\rho)}$ which determines the number of measurement settings in the theorem above. When the state $\rho$ is the equal eigenvalue state $\rho_{0}$, we get $\lambda_{\min }\left(\rho_{0}\right)=1 / r$ and we recover the rate presented in [31].

It was noted in [31] that deriving a concentration in the MSE via a concentration of Fisher average $I(\rho \mid \mathcal{S})$ provides a pessimistic estimate of the number of settings needed. Simulations in [31] demonstrated that the MSE concentrates for a much smaller number of settings $k$ than predicted. In the theorem presented above, we note that the dependence of the number of settings on the minimum eigenvalue of $\rho$ suggests a lack of concentration as $\lambda_{\min }(\rho)$ is made arbitrarily small. The number of required settings $k \rightarrow \infty$ in the limit that $\lambda_{\min }(\rho) \rightarrow 0$. This is because the maximum eigenvalue of the Fisher information $I(\rho \mid \mathbf{s})$ over all settings $\mathbf{s}$ becomes arbitrarily large when the rank-r state $\rho$ is arbitrarily close to being pure. However, as we shall demonstrate, this does not reflect the behaviour of the MSE concentration. Instead of deriving a concentration about $\bar{I}(\rho)$ as in the above theorem, we derive a useful upper bound for the MSE that is independent of the spectrum of the state.

Theorem 2. Let $\mathcal{S}=\left\{\mathbf{s}_{1}, \ldots, \mathbf{s}_{k}\right\}$ be a design with randomly, uniformly distributed measurement bases. Let $I_{\mathcal{S}}:=I(\rho \mid \mathcal{S})$ be the associated Fisher information evaluated at $\rho$. For a sufficiently small $\epsilon \geq 0$, the following inequality holds

$$
\operatorname{Tr}\left[I(\rho \mid \mathcal{S})^{-1} G_{F}\right] \leq 2(1+\epsilon) \frac{r+1}{r} D
$$

with probability $1-\delta$, provided that the number of measurements performed is $k=$ $C_{1}(r+1) \log (2 D / \delta)$, with $D=2 r d-r^{2}-1$ the dimension of the space of rank-r states, and $C_{1}=4\left(\log 2 / \epsilon^{2}\right)$.

The upper bound is roughly twice the number of unknown parameters, and although not optimal, it demonstrates that the MSE concentrates below a meaningful threshold given a fixed $O(r \log D)$ scaling in the number of settings. The proof of this theorem follows now. A key element in the proof is to overcome the potential unboundedness of the maximum eigenvalue of $I(\rho \mid \mathbf{s})$. This is done by bounding $I(\rho \mid \mathbf{s})$ from below over all possible settings $\mathbf{s}$ by matrices whose spectrums are well behaved. This in turn gives us an upper bound for the inverse of the sum $I(\rho \mid \mathcal{S})^{-1}$. 
To this end, we define a new state $\tilde{\rho}$ such that over all possible settings s, we have the following inequality in the Fisher matrices

$$
I(\rho \mid \mathbf{s}) \geq \frac{1}{2} I(\tilde{\rho} \mid \mathbf{s}) .
$$

The state $\tilde{\rho}$ is defined to be $\tilde{\rho}:=\left(\rho+\rho_{0}\right) / 2$, where $\rho_{0}$ is the rank-r state with equal $1 / r$ eigenvalues, and the same eigenvectors as $\rho$. It is easy to see that $\tilde{\rho}$ has eigenvalues bounded between $(1+1 / r) / 2$ and $1 / 2 r$, and has the same eigenvectors as $\rho$ by construction. The above inequality then follows from the fact that $\rho \leq 2 \tilde{\rho}$, and from the definition of the Fisher information matrix (5). For any given measurement design $\mathcal{S}=\left\{\mathbf{s}_{1}, \ldots \mathbf{s}_{k}\right\}$, this inequality in the Fisher matrices implies that $I(\rho \mid \mathcal{S}) \geq I(\tilde{\rho} \mid \mathcal{S}) / 2$. Since the matrix $I(\tilde{\rho} \mid \mathbf{s})$ has eigenvalues that are well behaved over all possible settings $\mathbf{s}$, we can use Theorem 1 to meaningfully bound the deviation $G_{F}^{-1 / 2} I(\tilde{\rho} \mid \mathcal{S}) G_{F}^{-1 / 2}$ from its mean. In fact, we get that for a sufficiently small $\epsilon \geq 0$, the following inequality holds

$$
(1-\epsilon) \operatorname{Tr}\left[\bar{I}(\tilde{\rho})^{-1} G_{F}\right] \leq \operatorname{Tr}\left[I(\tilde{\rho} \mid \mathcal{S})^{-1} G_{F}\right] \leq(1+\epsilon) \operatorname{Tr}\left[\bar{I}(\tilde{\rho})^{-1} G_{F}\right]
$$

with probability $1-\delta$, provided that the number of settings $k=C_{1}(r+1) \log (2 D / \delta)$. The upper bound in the equation above, combined with the inequality $I(\rho \mid \mathcal{S}) \geq I(\tilde{\rho} \mid \mathcal{S}) / 2$ gives the stated upper bound

$$
\operatorname{Tr}\left[I(\rho \mid \mathcal{S})^{-1} G_{F}\right] \leq 2(1+\epsilon) \operatorname{Tr}\left[\bar{I}(\tilde{\rho})^{-1} G_{F}\right] \leq 2(1+\epsilon) \frac{r+1}{r} D .
$$

Theorem 2 derives a uniform upper bound for all $r a n k-r$ states irrespective of the eigenvalue spectrum. This demonstrates that sensible bounds exist in the limit of $\lambda_{\min }(\rho) \rightarrow 0$ for a finite number of measurement settings $k$. It is clear that the divergence of the maximum eigenvalue $\max _{\mathbf{s}} \lambda_{\max }(I(\rho \mid \mathbf{s}))$ as $\lambda_{\min }(\rho) \rightarrow 0$ does not cause a similar divergence in the MSE. Therefore Theorem 1 does not sensibly define a rate for the required number of measured settings $k$ in the limit $\lambda_{\min }(\rho) \rightarrow 0$.

Although Theorem 2 derives a uniform upper bound for the MSE, it does not demonstrate a concentration in the MSE. However, for the simplified model for a rank - 2 qubit state, we show that a concentration in the MSE does in fact hold in the limit $\lambda_{\min }(\rho) \rightarrow 0$.

\section{The Single Qubit Model}

In this section we work with the simple model of a rank -2 qubit state to show that a concentration in the MSE about its optimal holds in the limit $\lambda_{\min }(\rho) \rightarrow 0$ without requiring the sum $I(\rho \mid \mathcal{S})$ to concentrate about $\bar{I}$.

Lemma 2. Let $\rho$ be a single qubit rank -2 state, and let $\mathcal{S}=\left\{\mathbf{s}_{1}, \ldots, \mathbf{s}_{k}\right\}$ be a uniformly random measurement design. Let $I_{\mathcal{S}}:=I(\rho \mid \mathcal{S})$ be the associated Fisher information, and let $\bar{I}(\rho)$ be the mean Fisher information over all possible measurement bases. For any $\epsilon>0$, there exists a finite $k$ such that the following inequality holds for all $\rho$ with high probability

$$
\operatorname{Tr}\left[I(\rho \mid \mathcal{S})^{-1} G_{F}\right] \leq(1+\epsilon) \operatorname{Tr}\left[\bar{I}(\rho)^{-1} G_{F}\right] .
$$

In order to investigate the behaviour of the MSE concentration as the spectrum is varied, we consider the generic state $\rho:=\lambda_{1}|0\rangle\left\langle 0\left|+\lambda_{2}\right| 1\right\rangle\langle 1|$ diagonal in its eigenbasis. We consider the 

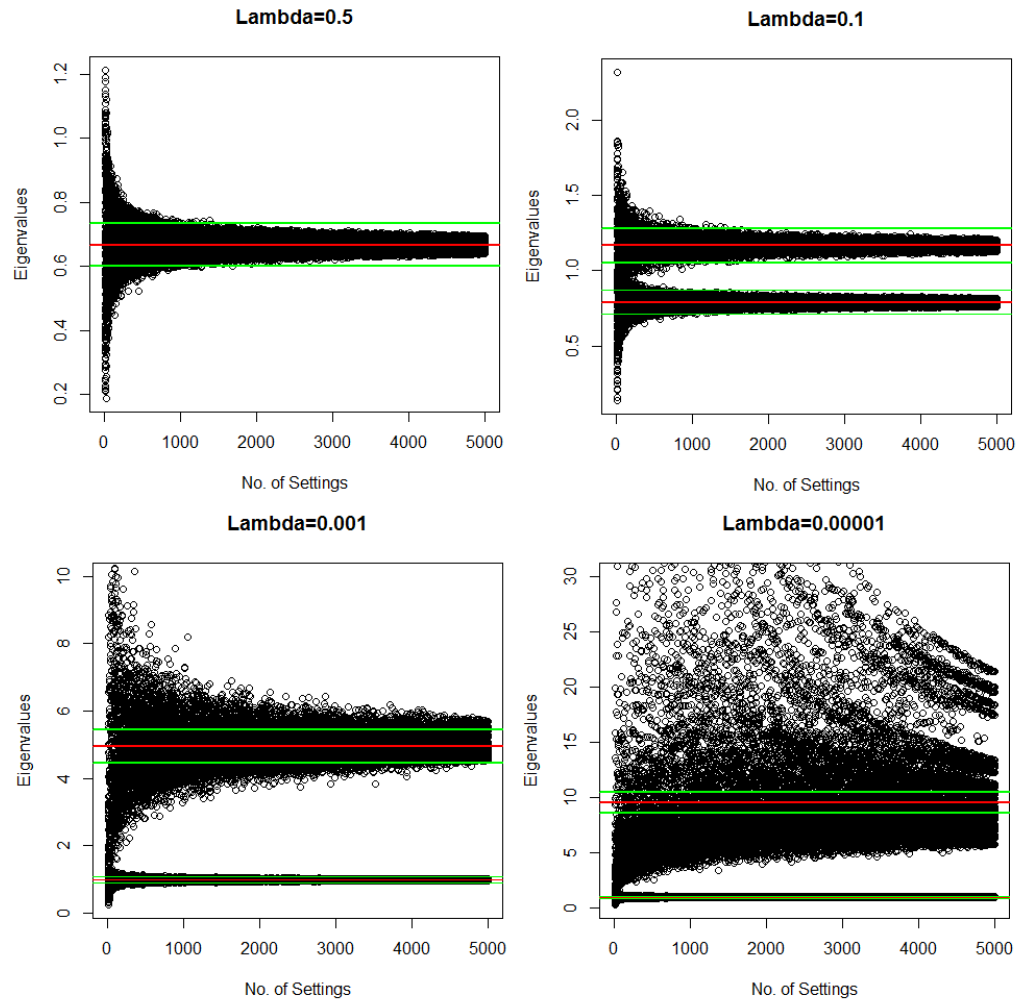

Figure 1. Plots of the eigenvalues of $G_{F}^{-1 / 2} I(\rho \mid \mathcal{S}) G_{F}^{-1 / 2}$ for various $k$ random settings. We chose 40 random single qubit states for each of the four values of $\lambda_{2}$. The red line indicates the eigenvalues of $\bar{I}(\rho) / 2$, with the green marking the $(1 \pm \epsilon) \bar{I} / 2$ deviations $(\epsilon=0.1)$. We observe that as the state becomes purer, the number of settings needed for concentration increases, and in the limit $\lambda_{2} \rightarrow 0$ there is a lack of concentration of the largest eigenvalue.

same local parametrisation as in the previous sections and denote $\boldsymbol{\theta}:=\left(\lambda_{2}, \operatorname{Re} \rho_{1,2}, \operatorname{Im} \rho_{1,2}\right)$. The measurement design consists of random, uniformly distributed measurement bases, and without loss of generality we set the projection vector corresponding to the +1 outcome for a given setting $\mathbf{s}$ as:

$$
\left|e_{\mathbf{s}}^{+1}\right\rangle:=\cos \frac{\phi}{2}|0\rangle+e^{i \omega} \sin \frac{\phi}{2}|1\rangle \quad 0 \leq \phi \leq \pi, 0 \leq \omega \leq 2 \pi
$$

The orthogonal vector corresponds to the -1 outcome. Therefore, the probabilities $p(\mathbf{o} \mid \mathbf{s})$ corresponding to the two outcomes are $p(+1 \mid \mathbf{s})=\left(1-\lambda_{2}\right) \cos ^{2} \frac{\phi}{2}+\lambda_{2} \sin ^{2} \frac{\phi}{2}$ and $p(-1 \mid \mathbf{s})=$ $\left(1-\lambda_{2}\right) \sin ^{2} \frac{\phi}{2}+\lambda_{2} \cos ^{2} \frac{\phi}{2}$. From equation (5), we evaluate the elements of the Fisher 

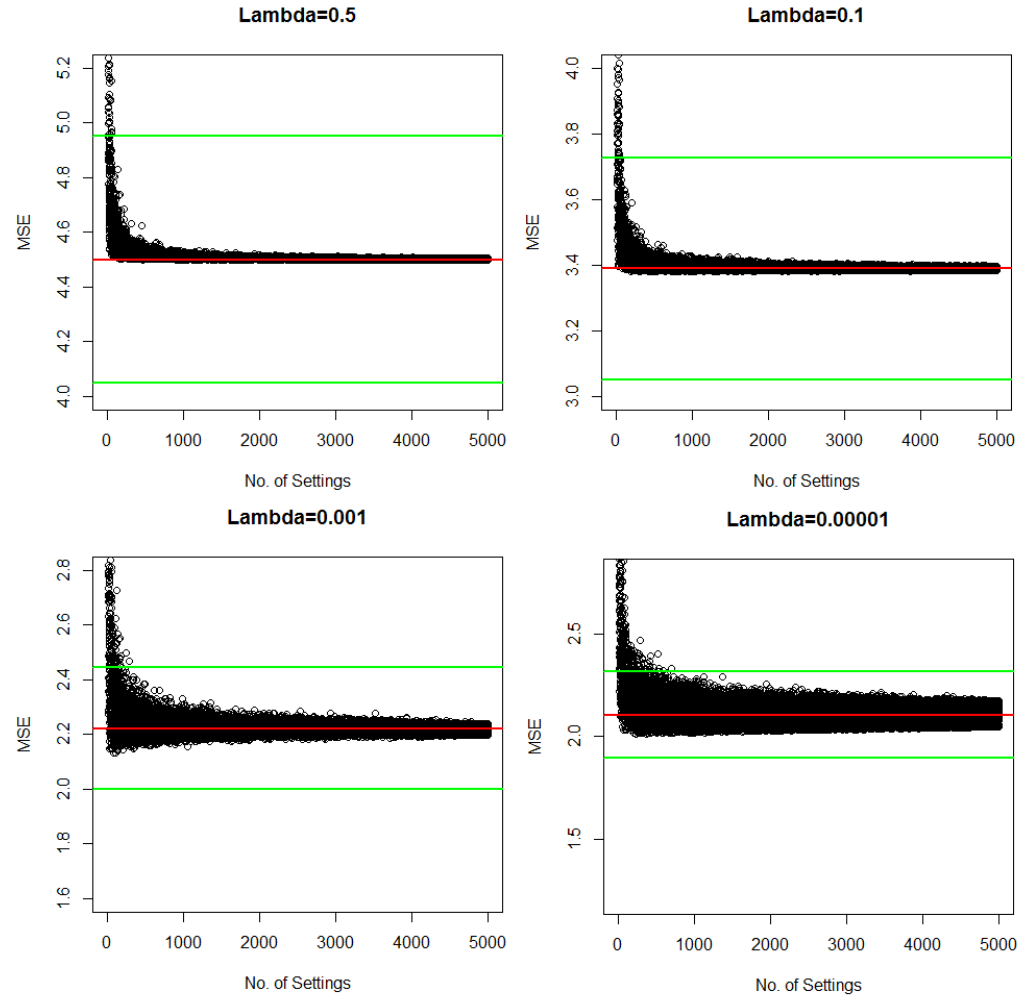

Figure 2. Plots of the MSE $\operatorname{Tr}\left[I(\rho \mid \mathcal{S})^{-1} G_{F}\right]$ for various $k$ random settings. We chose 40 random single qubit states for each of the four values of $\lambda_{2}$. The red line indicates the theoretical optimal MSE $\operatorname{Tr}\left[\bar{I}^{-1} G_{F}\right]$, with the green marking the $(1 \pm \epsilon) \operatorname{Tr}\left[\bar{I}^{-1} G_{F}\right]$ deviations $(\epsilon=0.1)$. It is easier to observe concentration in the MSE, despite a lack of concentration of $I(\rho \mid \mathcal{S})$ (see Figure 1). Although the number of settings needed for concentration within a prescribed relative error increases with a decrease in $\lambda_{2}$, there is a limiting value of $k$ as $\lambda_{2} \rightarrow 0$ (see text).

information matrix for a given random measurement setting $\mathbf{s}$.

$$
\begin{aligned}
I(\rho \mid \mathbf{s}) & =\left(\begin{array}{ccc}
I^{d d}(\rho \mid \mathbf{s}) & I^{r d}(\rho \mid \mathbf{s}) & I^{i d}(\rho \mid \mathbf{s}) \\
I^{r d}(\rho \mid \mathbf{s}) & I^{r r}(\rho \mid \mathbf{s}) & I^{r i}(\rho \mid \mathbf{s}) \\
I^{i d}(\rho \mid \mathbf{s}) & I^{r i}(\rho \mid \mathbf{s}) & I^{i i}(\rho \mid \mathbf{s})
\end{array}\right) \\
& =\frac{2}{1-\cos ^{2}(\phi)\left(1-2 \lambda_{2}\right)^{2}}\left(\begin{array}{ccc}
2 \cos ^{2}(\phi) & -\cos (\omega) \sin (2 \phi) & \sin (\omega) \sin (2 \phi) \\
-\cos (\omega) \sin (2 \phi) & 2 \cos ^{2}(\omega) \sin ^{2}(\phi) & -\sin (2 \omega) \sin ^{2}(\phi) \\
\sin (\omega) \sin (2 \phi) & -\sin (2 \omega) \sin ^{2}(\phi) & 2 \sin ^{2}(\omega) \sin ^{2}(\phi)
\end{array}\right)
\end{aligned}
$$

As before $\mathcal{S}$ is the set of $k$ randomly chosen settings $\mathbf{s}$, and as the settings in $\mathcal{S}$ are independent, the Fisher information matrices $I(\rho \mid \mathbf{s})$ are independent.

The concentration of the quantity $I(\rho \mid \mathcal{S}):=\frac{1}{k} \sum_{\mathbf{s} \in \mathcal{S}} I(\rho \mid \mathbf{s})$ around the mean Fisher matrix $\bar{I}(\rho)$ is given by Theorem 1 . We recall that the number of settings $k$ required to bound the 


\begin{tabular}{|c|c|c|}
\hline Fisher Element & Mean & Range \\
\hline$I^{d d}$ & $\frac{2 \ln \left[2\left(1-\lambda_{2}\right)\right]-2 \ln \left[2 \lambda_{2}\right]}{\left(1-2 \lambda_{2}\right)^{3}}-\frac{4}{\left(1-2 \lambda_{2}\right)^{2}}$ & {$\left[0, \frac{1}{\lambda_{2}\left(1-\lambda_{2}\right)}\right]$} \\
\hline$I^{r d}, I^{i d}$ & 0 & {$\left[\frac{-2}{\sqrt{\lambda_{2}\left(1-\lambda_{2}\right)}}, \frac{2}{\sqrt{\lambda_{2}\left(1-\lambda_{2}\right)}}\right]$} \\
\hline$I^{r i}$ & 0 & {$[-2,2]$} \\
\hline$I^{r r}, I^{i i}$ & $\frac{\ln \left[2\left(1-\lambda_{2}\right)\right]-\ln \left[2 \lambda_{2}\right]}{\left(1-2 \lambda_{2}\right)}-\frac{\bar{I}^{d d}}{2}$ & {$[0,4]$} \\
\hline
\end{tabular}

Table 1. The mean and range of the elements of the Fisher matrix $I(\rho \mid \mathbf{s})$ as functions of $\lambda_{2}$. Note that the expressions for the means in the table above are valid for all $\lambda_{2}<0.5$. When $\lambda_{2}=0.5$, then all diagonal elements $\bar{I}^{r r / d d / i i}$ have the same value of $4 / 3$.

deviation from its mean $\bar{I}(\rho)$ depends on the ratio of the eigenvalues

$$
\frac{\mu_{\max }}{\mu_{\min }}:=\frac{\max _{\mathbf{s}} \lambda_{\max }\left(G_{F}^{-1 / 2} I(\rho \mid \mathbf{s}) G_{F}^{-1 / 2}\right)}{\lambda_{\min }\left(G_{F}^{-1 / 2} \bar{I}(\rho) G_{F}^{-1 / 2}\right)} .
$$

For the simple qubit model these can be explicitly evaluated. For settings with $\phi=0$, the maximum eigenvalue is $\frac{1}{2 \lambda_{2}\left(1-\lambda_{2}\right)}$. This implies that $\mu_{\max } \geq \frac{1}{4 \lambda_{2}\left(1-\lambda_{2}\right)}$. This value is a contribution from the $I^{d d}$ element of the Fisher matrix, and tends to infinity as $\lambda_{2} \rightarrow 0$. The minimum eigenvalue $\mu_{\min }$ is a contribution from the $\bar{I}^{r r}$ and the $\bar{I}^{i i}$ term, and tends to a limiting value of 1 when $\lambda_{2} \rightarrow 0$. The explicit expressions can be found in Table 1 . Taken together this implies that the ratio becomes unbounded as $\lambda_{2} \rightarrow 0$. This is precisely the difficultly characterised in the previous section, and is illustrated in Figure 1, where we plot the eigenvalues of the sum $G_{F}^{-1 / 2} I(\rho \mid \mathcal{S}) G_{F}^{-1 / 2}$ for various values of $\lambda_{2}$ and choices of measurement designs $\mathcal{S}$.

However we are not interested in the concentration of the Fisher matrix itself, but rather the quantity $\operatorname{Tr}\left[I(\rho \mid \mathcal{S})^{-1} G_{F}\right]$, and in Figure 2 it is seen that the MSE exhibits clear concentration about the optimal. Although the number of settings needed for the MSE to be within $(1 \pm \epsilon)$ of the optimal is seen to increase for smaller values of $\lambda_{2}$, we shall show that there exists a limiting value of $k$ as $\lambda_{2} \rightarrow 0$. To demonstrate this, we consider the concentration of the individual Fisher elements, and directly bound the deviation of $\operatorname{Tr}\left[I(\rho \mid \mathcal{S})^{-1} G_{F}\right]$ from its optimal.

It is clear from Table 1 that the Fisher matrix elements $I^{r r}, I^{i i}, I^{r i}$ have bounded means and spread even in the limit $\lambda_{2} \rightarrow 0$. Their sums can therefore be shown to concentrate around their means using one of several concentration inequalities. For example, we apply Hoeffding's inequality below.

Fact 1. Let $X_{1}, \ldots, X_{k}$ be independent random variables such that each $X_{i}$ is bounded as $a \leq X_{i} \leq b$, and let $\mu:=\mathbb{E}[X]$. Let $S_{k}:=\frac{1}{k} \sum_{i}^{k} X_{i}$, and $C:=b-a$, then for any $t \geq 0$ and $\tau>0$ the following inequalities hold,

(i) Hoeffding's inequality : $\left.\mathbb{P}\left(\left|S_{k}-\mu\right|\right) \geq t\right) \leq 2 e^{-2 k t^{2} / C^{2}}$

(ii) Markov's Inequality: $\mathbb{P}\left(\left|S_{k}\right| \geq \tau\right) \leq \frac{\mathbb{E}|X|}{\tau}$

(iii) Chebyshev's Inequality: $\mathbb{P}\left(\left|S_{k}-\mu\right| \geq \tau\right) \leq \frac{\operatorname{Var}(X)}{\tau^{2}}$ 
From Table 1 we see that $C=4$ for the $I^{r r}, I^{i i}, I^{r i}$ matrix elements. Thus we derive that for any $t \geq 0$, their empirical means are within $\pm t$ of the true value with probability $(1-\delta)$, provided that the number of settings $k \geq\left(8 / t^{2}\right) \ln (2 / \delta)$. Therefore the concentration for these elements is well behaved in the limit $\lambda_{2} \rightarrow 0$. While the same inequality can be applied to $I^{r d}, I^{i d}$ matrix elements when $\lambda_{2}$ is away from zero, it fails in the limit $\lambda_{2} \rightarrow 0$ because their ranges become infinite. However, we make a 'weak law of large numbers' argument to show that even in this limit, there exists a finite but 'sufficiently large' $k$, such that $I^{r d}(\rho \mid \mathcal{S})$ and $I^{i d}(\rho \mid \mathcal{S})$ concentrate around their mean.

The key point is that the random variables $I^{r d}, I^{i d}$ remain absolutely integrable in limit $\lambda_{2} \rightarrow 0$. This is combined with a truncation trick, to show that although the range of these variables in unbounded in the limit, for 'sufficiently large' $k$ their empirical means converge in probability to their expected value. We follow the argument presented in [42] to demonstrate this. The idea of the truncation method is to split the random variable $I^{r d}$ as

$$
\begin{aligned}
I^{r d} & :=I_{\leq T}^{r d}+I_{>T}^{r d} \\
& =I^{r d} \mathbb{1}\left(\left|I^{r d}\right| \leq T\right)+I^{r d} \mathbb{1}\left(\left|I^{r d}\right|>T\right),
\end{aligned}
$$

with $T$ being a 'truncation parameter' that is chosen appropriately. We shall not be interested in the actual value of $T$, but endeavour only to show that such a method demonstrates the existence of a finite $k$ for which $I^{r d}$ converges in probability to zero. We similarly split the sum

$$
I^{r d}(\rho \mid \mathcal{S})=\frac{1}{k} \sum_{i=1}^{k}\left[I_{\leq T}^{r d}\left(\rho \mid \mathbf{s}_{i}\right)+I_{>T}^{r d}\left(\rho \mid \mathbf{s}_{i}\right)\right]=: I_{\leq T}^{r d}(\rho \mid \mathcal{S})+I_{>T}^{r d}(\rho \mid \mathcal{S})
$$

We now bound these two sums using different inequalities. Since the random variable is absolutely integrable even in the limit $\lambda_{2} \rightarrow 0$, we can always choose the truncation parameter $T$ such that $\mathbb{E}\left|I_{>T}^{r d}\right|$ is made small, say some $\delta_{2}>0$, so that from Markov's inequality (Fact 1) we get

$$
\mathbb{P}\left(\left|I_{>T}^{r d}(\rho \mid \mathcal{S})\right| \geq \tau\right) \leq \frac{\delta_{2}}{\tau}
$$

The variable $I_{\leq T}^{r d}$ has bounded spread by construction, and therefore has bounded variance. This allows us to use Chebyshev's inequality (Fact 1), from which we see that

$$
\mathbb{P}\left(\left|I_{\leq T}^{r d}(\rho \mid \mathcal{S})\right| \geq \tau\right) \leq \frac{\operatorname{Var}\left(I_{\leq T}^{r d}\right)}{k \tau^{2}}
$$

where we use the fact that $\mathbb{E}\left(I_{\leq T}^{r d}\right)=0$, since the distribution is symmetric about zero. Clearly $\operatorname{Var}\left(I_{\leq T}^{r d}\right)$ is bounded, and there exists a finite $k$ such that (20) and (21) together imply

$$
\mathbb{P}\left(\left|I^{r d}(\rho \mid \mathcal{S})\right| \geq \tau\right) \leq \frac{\delta_{2}}{\tau}+\frac{1}{\tau^{2}} .
$$

The term $\delta_{2}$ can be made arbitrarily small by choosing $T$ appropriately, which demonstrates that the sum converges in probability to zero for some finite, but 'sufficiently large' $k$. Although the above argument was demonstrated with $I^{r d}(\rho \mid \mathcal{S})$, the same holds for $I^{i d}(\rho \mid \mathcal{S})$. This leaves the term $I^{d d}(\rho \mid \mathcal{S})$, which due to the non-integrability, infinite mean and range of $I^{d d}$ in the limit $\lambda_{2} \rightarrow 0$, does not concentrate around any finite value. The term $I^{d d}$ contributes the maximum eigenvalue of the Fisher matrix over all settings $\mathbf{s}$, and as mentioned earlier its divergence in the limit $\lambda_{2} \rightarrow 0$ is why a concentration inequality of the form of Theorem 1 
does not hold. Collecting the individual bounds for the other matrix elements, we have that for any value of $\lambda_{2}$ there exists a finite $k$ for which with large probability, the matrix sum $I(\rho \mid \mathcal{S})$ has elements

$$
\left(\begin{array}{ccc}
\sum_{i=1}^{k} I_{i}^{d d} / k & {[-\tau,+\tau]} & {[-\tau,+\tau]} \\
{[-\tau,+\tau]} & {[\mu-t, \mu+t]} & {[-t,+t]} \\
{[-\tau,+\tau]} & {[-t,+t]} & {[\mu-t, \mu+t]}
\end{array}\right)
$$

where $\mu:=\mathbb{E}\left(I^{r r / i i}\right)$. We can now explicitly evaluate $\operatorname{Tr}\left[I(\rho \mid \mathcal{S})^{-1} G_{F}\right]$, making the simplifying assumption that $k$ is large enough to ignore terms quadratic in the off-diagonal elements, i.e, in $\tau$ and $t$. Going through the calculation, we get that provided $\sum_{i=1}^{k} I_{i}^{d d} / k>$ 1 ,

$$
\operatorname{Tr}\left[I(\rho \mid \mathcal{S})^{-1} G_{F}\right] \leq \frac{2 k}{\sum_{i=1}^{k} I_{i}^{d d}}+\frac{4}{\mu-t} .
$$

In order to show that the MSE is close to optimal as in Lemma 2, we require that the term on the right in the above equation is smaller than $(1+\epsilon) \operatorname{Tr}\left[\bar{I}(\rho)^{-1} G_{F}\right]$. That is, for some $\epsilon>0$,

$$
\frac{k}{\sum_{i=1}^{k} I_{i}^{d d}}+\frac{2}{\mu-t} \leq(1+\epsilon)\left[\frac{1}{\bar{I}^{d d}}+\frac{2}{\mu}\right] \text {. }
$$

When $\lambda_{2}$ is sufficiently large, the random variable $I^{d d}$ is bounded and therefore the sum $\sum_{i=1}^{k} I_{i}^{d d} / k$ concentrates about its mean. In the limit $\lambda_{2} \rightarrow 0$ however, $1 / \bar{I}^{d d} \rightarrow 0$, which implies that the sum $\sum_{i=1}^{k} I_{i}^{d d} / k$ does not need to concentrate about its (infinite) mean, but only needs to be larger than a value dependent on $\epsilon$ and $t$. In the limit $\lambda_{2} \rightarrow 0$, the Fisher element $I^{d d}$ has a limiting distribution which can be explicitly evaluated. Setting $\lambda_{2}=0$ in (16) we have that $I^{d d}=4 \cot ^{2} \phi$. Inverting this we get $\phi=\cot ^{-1}\left(\sqrt{I^{d d}(\phi)} / 2\right)$, and as the projection vectors are drawn uniformly over the unit sphere, $\phi$ is distributed as $f_{\Phi}(\phi) d \phi=\sin \phi d \phi$. Performing a change of variables then gives the limiting distribution $f_{I}\left(I^{d d}\right) d I^{d d}=\frac{2}{\sqrt{I^{d d}}\left(I^{d d}+4\right)^{3 / 2}} d I^{d d}$. From this distribution and the truncation method it is easy to show that for any value $C$, there exists a finite number of settings $k$ such that $\sum I^{d d} / k>C$. This implies that for a given $\epsilon>0$, and for all values of $\lambda_{2} \in(0,0.5]$ there always exists a finite number of settings $k$ such that the required concentration holds.

\subsection{Estimation Error in Terms of Quantum Infidelity}

In this section we consider the problem of 'compressive' state estimation in terms of a different metric, the quantum infidelity

$$
1-F(\hat{\rho}, \rho)=1-\operatorname{Tr}(\sqrt{\sqrt{\rho} \hat{\rho} \sqrt{\rho}})^{2}
$$

As briefly hinted at in the introduction, a local expansion of this metric is not quadratic uniformly over all states. In particular for states that are well in the interior of the state space the expansion is locally quadratic, while for states with eigenvalues that are close to zero, the infidelity becomes linear [39]. This linear expansion highlights the sensitivity of the infidelity to misestimation of small eigenvalues, and we show that in our setup with uniformly random basis measurements, 'compressive' estimation for all states in the sense of Lemma 2 does 
not hold for this metric. To demonstrate this we continue considering the single qubit model from the previous section. We derive a theorem for the concentration of the mean infidelity (MINF) for states well within the Bloch sphere, and then demonstrate a lack of concentration for nearly pure states. As before, we consider the state $\rho=\operatorname{Diag}\left(1-\lambda_{2}, \lambda_{2}\right)$ diagonal in its eigenbasis. For qubits, the infidelity can be expressed as [43]

$$
1-F(\hat{\rho}, \rho)=1-\operatorname{Tr}(\hat{\rho} \rho)-2 \sqrt{\operatorname{det} \hat{\rho} \cdot \operatorname{det} \rho} .
$$

A Taylor expansion of the infidelity about $\rho$ demonstrates that for states within the Bloch sphere (i.e. $\lambda_{2}$ is well away from zero), the infidelity is locally quadratic in the (local) parameters

$$
1-F\left(\rho_{\boldsymbol{\theta}}, \rho_{\boldsymbol{\theta}+\boldsymbol{\delta} \boldsymbol{\theta}}\right)=(\boldsymbol{\delta} \boldsymbol{\theta})^{T} G_{I N F}(\boldsymbol{\delta} \boldsymbol{\theta})+O\left(\|\boldsymbol{\delta} \boldsymbol{\theta}\|^{3}\right),
$$

where $G_{I N F}=\operatorname{Diag}\left(1 / 2 \lambda_{2}\left(1-\lambda_{2}\right), 2,2\right)$ is the weight matrix reproducing the infidelity. In general for states of arbitrary dimension that have eigenvalues away from zero, the local expansion remains quadratic [39], and a concentration of the MINF is readily established using the techniques in the previous sections. Here we formulate this concentration for the single qubit state considered. Combining the above local expansion with the asymptotic normality of efficient estimators (6), the MINF is given by an expression similar to (8)

$$
\mathrm{MINF}:=\mathbb{E}(1-F(\hat{\rho}, \rho)) \approx \frac{1}{N} \operatorname{Tr}\left(I(\rho \mid \mathcal{S})^{-1} G_{I N F}\right) .
$$

A concentration of this error term can be demonstrated using the same tools used to establish Theorem 1. Concretely, we derive the following theorem.

Lemma 3. Let $\mathcal{S}=\left\{\mathbf{s}_{1}, \ldots, \mathbf{s}_{k}\right\}$ be a design with randomly, uniformly distributed measurement bases. Let $I_{\mathcal{S}}:=I(\rho \mid \mathcal{S})$ be the associated Fisher information, and let $\bar{I}$ be the mean Fisher information over all possible bases, both calculated at the single qubit state $\rho$. For a sufficiently small $\epsilon \geq 0$, the following inequality holds

$$
(1-\epsilon) \operatorname{Tr}\left[\bar{I}^{-1} G_{I N F}\right] \leq \operatorname{Tr}\left[I_{\mathcal{S}}^{-1} G_{I N F}\right] \leq(1+\epsilon) \operatorname{Tr}\left[\bar{I}^{-1} G_{I N F}\right]
$$

with probability $1-\delta$, provided that the number of measurements performed is $k=$ $\frac{C_{2}}{\lambda_{2}\left(1-\lambda_{2}\right)} \log \left(\frac{2 D}{\delta}\right)$, with $D=3$ the dimension of the space of rank-2 qubit states.

Where $C_{2}$ is a constant depending on $\epsilon$ that can be arbitrarily set. Due to the dependence of the number of settings $k$ on the minimum eigenvalue of the true state, the above lemma sensibly demonstrates concentration only when $\lambda_{2}$ is away from zero. This is similar to the dependence of the number of settings on $\lambda_{\min }(\rho)$ in Theorem 1 .

In the case of the MSE we demonstrated that for qubits, concentration does occur as $\lambda_{2} \rightarrow 0$, even if Theorem 1 does not hold in this limit. However, a similar concentration of the MINF for qubits in this limit does not occur. To show this, we first notice that in the limit $\lambda_{2} \rightarrow 0$ the local expansion of the infidelity becomes linear in the leading order

$$
1-F\left(\rho_{\boldsymbol{\theta}}, \rho_{\theta+\boldsymbol{\delta} \boldsymbol{\theta}}\right)=\left|\delta \theta^{d}\right|+O\left(\|\boldsymbol{\delta} \boldsymbol{\theta}\|^{2}\right) .
$$

Clearly, for estimates $\hat{\rho}$ in the local neighbourhood of the pure state $\rho=|0\rangle\langle 0|$, the MINF is no longer given by the quadratic expression as in (29), but is $\mathbb{E}(1-F(\hat{\rho}, \rho))=$ $\mathbb{E}\left(\hat{\theta}^{d}\right)=\mathbb{E}(\langle 1|\hat{\rho}| 1\rangle)$. Since the dominant error term is linear in the diagonal element of the estimate (in the eigenbasis of the true state), we note that the infidelity is highly sensitive to the misestimation of small eigenvalues [39]. The errors in the estimation of the 'rotation 


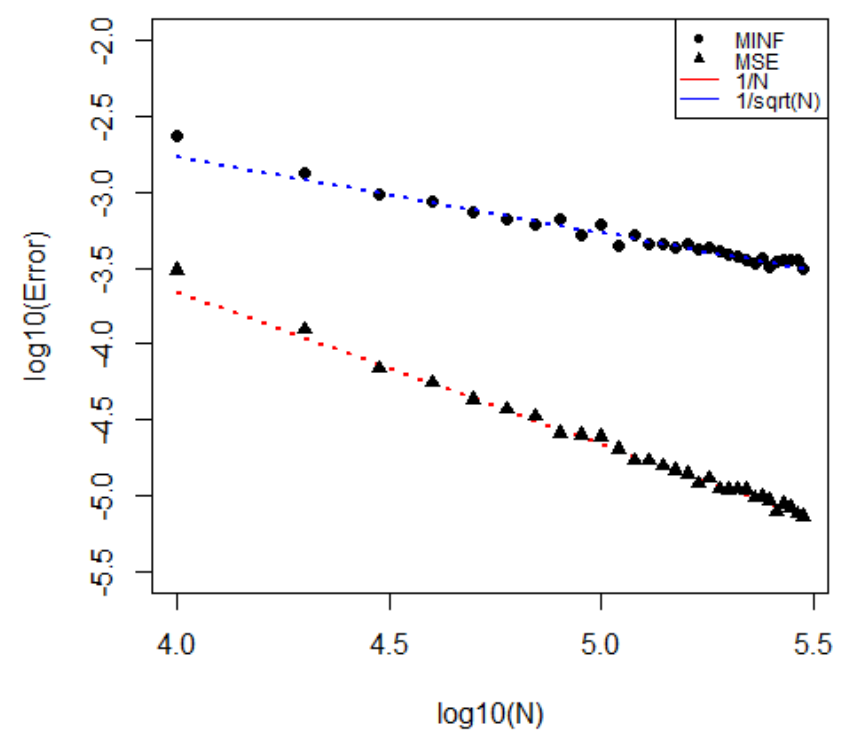

Figure 3. Plots of the MINF and MSE of the maximum likelihood estimate of a randomly chosen single qubit pure state and random basis measurements. The total number of samples of the state is $N=k \times m$, where $k$ is the number of settings measured and $m=1000$ is the number of repetitions per setting. The number of random basis measured $k$ is varied between 10 and 300. The expected error is approximated over 300 different choices of $k$ randomly chosen settings. The MSE demonstrates a $O(1 / N)$ scaling, while for the same estimates the MINF scales as $O(1 / \sqrt{N})$.

parameters' $\theta^{r}, \theta^{i}$ however remain quadratic, and therefore exhibit a $O(1 / N)$ scaling as in the previous sections. As the interesting contribution to the infidelity is from the estimation errors of the eigenvalue, we consider a simplified single parameter model and assume that only $\theta^{d}$ is unknown. When the number of repetitions $m$ in a setting $\mathbf{s}$ is sufficiently large, efficient estimators of $\theta^{d}$ from the outcomes of these measurements have an asymptotically Gaussian distribution $\sqrt{m}\left(\hat{\theta}^{d}-\theta^{d}\right) \approx N\left(0, \operatorname{Var}\left(\hat{\theta^{\mathrm{d}}}\right)\right)$. Therefore, in this asymptotic limit the MINF $\mathbb{E}(1-F(\hat{\rho}, \rho))$ is given by

$$
\mathbb{E}\left(\hat{\theta}^{d}\right)=\frac{1}{\sqrt{2 \sigma^{2} \pi}} \int_{0}^{\infty} \hat{\theta}^{d} \cdot \exp \left(-\frac{\left(\hat{\theta}^{\mathrm{d}}\right)^{2}}{2 \sigma^{2}}\right) \mathrm{d} \hat{\theta}^{\mathrm{d}}=\sqrt{\frac{2}{\pi}} \sigma
$$

where negative estimates of the parameter are set to zero to ensure that $\hat{\rho}$ is a density matrix, and the standard deviation $\sigma=\operatorname{Var}\left(\hat{\theta}^{\mathrm{d}}\right)^{1 / 2}$. From this asymptotic behaviour of efficient estimators we see that for a large number of repetitions $m$, the MINF scales as

$$
\mathbb{E}(1-F(\hat{\rho}, \rho)) \approx \sqrt{\frac{2}{\pi N}} \sqrt{I^{d d}(\rho \mid \mathcal{S})^{-1}},
$$

where the Fisher information $I^{d d}$ corresponding to the diagonal parameter is found in the previous section. From Table 1 and the discussion in the previous section, we know that in the 
limit $\lambda_{2} \rightarrow 0$ the mean Fisher information $\bar{I}^{d d}$ diverges. The Fisher information $I(\rho \mid \mathcal{S})$ for any finite sample of random measurements will therefore not concentrate within $1 \pm \epsilon$ of the optimal, implying a lack of concentration in the MINF. In the case of the Frobenius norm, in the limit $\lambda_{2} \rightarrow 0$ the dominant error terms contributing to the MSE correspond to the rotation parameters, and this fact ensures a concentration of the MSE even in the pure state limit. While for the infidelity we see that the dominant error terms comes from the estimation of the small eigenvalues, and a concentration of the MINF does not exist in the sense of Lemma 2. In general, the local expansion of the infidelity around any rank- $r$ state that is close to pure is linear in the diagonal terms of the estimate [39]. The MINF for such states therefore demonstrates a similar lack of concentration in the corresponding diagonal elements of the Fisher information matrix.

Furthermore, from (32) it is clear that with uniform random measurements the MINF scales as $O(1 / \sqrt{N})$ for states that are close to pure. While for states well within the Bloch sphere, Lemma 3 demonstrates a scaling of $O(1 / N)$. This poor scaling is observed in Figure 3, which plots the expected error in terms of the MINF and the MSE for pure states. As discussed in the previous sections, it is seen that the MSE scales as $O(1 / N)$ for all states, while it is clear from Figure 3 that the MINF demonstrates a $O(1 / \sqrt{N})$ scaling for pure states. This scaling has also been demonstrated for the closely related Bures distance error metric. In $[44,46]$, the minimax Bures error for estimators based on Pauli expectations is shown to scale as $O(1 / \sqrt{N})$. This poor scaling along with a lack of concentration is important as many quantum information tasks utilise states that are pure [39]. Several adaptive measurement protocols have been suggested and implemented [39, 47, 48, 49] to improve this scaling. The aim of such adaptive strategies is to make measurements that are close to the eigenbasis of the true state. In our qubit model, for measurements with angle $\phi$ smaller than $O(1 / \sqrt{N})$ the Fisher information $I^{d d}(\rho)$ scales as $O(N)$. From (32), this gives a $O(1 / N)$ scaling of the infidelity even in the limit $\lambda_{2} \rightarrow 0$.

\section{Conclusions}

In this paper we investigated the asymptotic behaviour of the error for an arbitrary optimal estimator in the random measurement setup. Specifically we looked at how the accuracy of efficient estimators depends on the measurement design and the state. We considered two distance measures, the Frobenius norm and the quantum infidelity. In the case of the Frobenius norm, we extended the concentration results in [31], and demonstrated that the MSE attains the optimal rate (up to a constant) with only $O(r \log D)$ random basis measurements for all states of rank $r$. Furthermore, to investigate the behaviour of the MSE concentration for states that are close to pure, we considered the model of a single qubit. We presented an argument to show that concentration in the MSE occurs for all qubit states, despite a lack of concentration in the Fisher information matrix for states close to the surface of the Bloch sphere.

It remains an open problem if a similar scaling of the MSE exists in the Pauli measurement setup used in standard multiple ions tomography. The application of the tools in the paper to the Pauli setup requires control of the eigenvalues in equation (9), specifically a lower bound on the minimum eigenvalue $\lambda_{\min }(\bar{I})$. Strong numerical evidence in [31] suggests that for random measurements the Fisher information may satisfy the required spectral properties. Concentration results for distances other than the Frobenius norm can be in principle derived using similar arguments as long as their local expansions are quadratic in the parameters (see (7)). However, for the infidelity (an important measure of error for quantum tomography), it is known that while the scaling is quadratic for states deep in the Bloch sphere, for states 
close to pure this scaling is linear in the parameters [39]. We demonstrated with a single qubit model that for such nearly pure states and random measurements, the mean infidelity (MINF) does not concentrate around the optimal for any finite number of settings. This implies a lack of 'compressive' recovery of such low rank states, and therefore by increasing the number of measurement settings one can always significantly decrease the corresponding estimation error.

The FIM has been an important tool in our investigation of both the MINF and the MSE. We noticed that the FIM fails to concentrate when one of the eigenvalues of the state approaches zero. Related work in establishing and using continuity relations of the Quantum Fisher information (QFI) [50,51] also shows a dependence on the smallest eigenvalues of the state, and therefore interesting behaviour occurs when eigenvalues approach zero. It is a possible direction for future research to see if our results about the concentration failure of the FIM are more deeply connected to the work in $[50,51]$.

\section{Appendix}

\section{Appendix A. Proof of Lemma 1}

Lemma. For any rank-r state $\rho$ with an arbitrary spectrum, and the rank-r state $\rho_{0}$ which has equal non-zero eigenvalues $1 / r$ and the same eigenvectors as $\rho$, the following inequality holds between their average Fisher information matrices, evaluated over all possible random measurement settings.

$$
\bar{I}\left(\rho_{0}\right) \leq \bar{I}(\rho)
$$

Proof. For a given random measurement setting $\mathbf{s}$, the probabilities of occurrence of an outcome $\mathbf{o}$ for the two states $\rho_{0}$ and $\rho$ are given by

$$
p_{0}(\mathbf{o} \mid \mathbf{s})=\sum_{i=1}^{r} \frac{1}{r}\left|\left\langle e_{\mathbf{s}}^{\mathbf{o}} \mid \lambda_{i}\right\rangle\right|^{2} \quad ; p_{\rho}(\mathbf{o} \mid \mathbf{s})=\sum_{i=1}^{r} \lambda_{i}\left|\left\langle e_{\mathbf{s}}^{\mathbf{o}} \mid \lambda_{i}\right\rangle\right|^{2}
$$

where $\lambda_{i}$ and $\left|\lambda_{i}\right\rangle$ are the eigenvalues and the eigenvectors of the state $\rho$ respectively. We now consider states $\rho^{\prime}$, that are constructed by permuting the $r$ non-zero eigenvalues $\lambda_{i}$ of the state $\rho$, while keeping the eigenvectors fixed. Let $\mathcal{P}$ denote the set of $r$ ! such permuted states. Averaging the probabilities $p_{\rho^{\prime}}(\mathbf{o} \mid \mathbf{s})$ over all the permuted states $\rho^{\prime} \in \mathcal{P}$ recovers the probability $p_{0}(\mathbf{o} \mid \mathbf{s})$ corresponding to the state with the uniform spectrum. That is,

$$
\frac{1}{|\mathcal{P}|} \sum_{\rho^{\prime} \in \mathcal{P}} p_{\rho^{\prime}}(\mathbf{o} \mid \mathbf{s})=p_{0}(\mathbf{o} \mid \mathbf{s})
$$

From the convexity of the function $f(x)=1 / x$ in the interval $(0,+\infty)$, the above equation together with Jensen's inequality implies,

$$
\frac{1}{p_{0}(\mathbf{o} \mid \mathbf{s})} \leq \frac{1}{|\mathcal{P}|} \sum_{\rho^{\prime} \in \mathcal{P}} \frac{1}{p_{\rho^{\prime}}(\mathbf{o} \mid \mathbf{s})}
$$


where we assumed that $p_{\rho^{\prime}}(\mathbf{o} \mid \mathbf{s})>0$ for all $\mathbf{o}$. From 5, we see that for a setting $\mathbf{s}$, the Fisher matrix in our parametrisation can be written as a sum of $d$ matrices

$$
I\left(\rho^{\prime} \mid \mathbf{s}\right)=\sum_{\mathbf{o}: p_{\rho^{\prime}}(\mathbf{o} \mid \mathbf{s})>0} \frac{1}{p_{\rho^{\prime}}(\mathbf{o} \mid \mathbf{s})}\left|V_{\mathbf{s}}^{\mathbf{o}}\right\rangle\left\langle V_{\mathbf{s}}^{\mathbf{o}}\right|
$$

where $\left|V_{\mathbf{s}}^{\mathbf{o}}\right\rangle \in \mathbb{R}^{D}$, with $D=2 r d-r^{2}-1$, are vectors that depend only on the measurement vectors $\left|e_{\mathbf{s}}^{\mathbf{o}}\right\rangle$, and the eigenvectors $\left|\lambda_{i}\right\rangle$ of the state. Since by construction the eigenvectors for all the states considered above are the same, together with A.3, we get for all settings $\mathbf{s}$

$$
\begin{aligned}
I\left(\rho_{0} \mid \mathbf{s}\right)=\sum_{\mathbf{o}: p_{0}(\mathbf{o} \mid \mathbf{s})>0} \frac{1}{p_{0}(\mathbf{o} \mid \mathbf{s})}\left|V_{\mathbf{s}}^{\mathbf{o}}\right\rangle\left\langle V_{\mathbf{s}}^{\mathbf{o}}\right| & \leq \frac{1}{|\mathcal{P}|} \sum_{\mathbf{o}} \sum_{\rho^{\prime} \in \mathcal{P}} \frac{1}{p_{\rho^{\prime}}(\mathbf{o} \mid \mathbf{s})}\left|V_{\mathbf{s}}^{\mathbf{o}}\right\rangle\left\langle V_{\mathbf{s}}^{\mathbf{o}}\right| \\
& =\frac{1}{|\mathcal{P}|} \sum_{\rho^{\prime} \in \mathcal{P}} I\left(\rho^{\prime} \mid \mathbf{s}\right) .
\end{aligned}
$$

The inequality holds for settings $\mathbf{s}$ such that $p_{\rho^{\prime}}(\mathbf{o} \mid \mathbf{s})>0$ for all "permuted" states $\rho^{\prime}$ and all outcomes $\mathbf{o}$, which holds with probability one under the Haar measure over settings. Since each $\rho^{\prime}$ is an unitary rotation of the state $\rho$, we arrive at the required inequality of the average Fisher matricies by integrating both sides of the above equation over all possible random measurement settings $\mathbf{s}$.

\section{Appendix B. Proof of Theorem 1}

The proof of this theorem is similar to the one presented in [31]. Here we present the important elements of the proof, and refer to [31] for details. As briefly mentioned in the main text of the paper, the proof of the theorem utilises the following matrix Chernoff bound [38], where the random matrices $X_{i}$ are given by $G_{F}^{-1 / 2} I\left(\rho \mid \mathbf{s}_{i}\right) G_{F}^{-1 / 2}$, with $\mathbf{s}_{i}$ random bases.

Theorem 3. (Matrix Chernoff Bound) Consider a finite sequence $X_{1}, \ldots, X_{k}$ of independent, random, positive matrices with dimension $D$, such that $\lambda_{\max }(X) \leq R$. For $\mathbb{E} X=M \geq \mu 1$ and $0 \leq \epsilon \leq \frac{1}{2}$,

$$
\mathbb{P}\left\{\frac{1}{k} \sum_{i=1}^{k} X_{i} \notin[(1-\epsilon) M,(1+\epsilon) M]\right\} \leq 2 D \cdot \exp \left(-k \cdot \frac{\epsilon^{2} \mu}{2 R \cdot \log 2}\right)
$$

We note that $G_{F}^{-1 / 2} I_{\mathcal{S}} G_{F}^{-1 / 2}$ is a sum of $k$ independent, random, positive matrices. In order to apply the above bound, we need to upper bound the largest eigenvalue of $G_{F}^{-1 / 2} I(\rho \mid \mathbf{s}) G_{F}^{-1 / 2}$ over all measurements, denoted $\mu_{\max }$. We also need to lower bound the smallest eigenvalue of the expected Fisher information $G_{F}^{-1 / 2} \bar{I}(\rho) G_{F}^{-1 / 2}$, denoted $\mu_{\text {min }}$. We will first derive these bounds and then obtain the result by applying the Chernoff bound. As in the text, we work with the local parametrisation

$$
\boldsymbol{\theta}=\left(\boldsymbol{\theta}^{(d)}, \boldsymbol{\theta}^{(r)}, \boldsymbol{\theta}^{(i)}\right)=\left(\rho_{2,2}, \ldots, \rho_{r, r} ; \operatorname{Re} \rho_{1,2}, \ldots, \operatorname{Re} \rho_{r, d} ; \operatorname{Im} \rho_{1,2}, \ldots, \operatorname{Im} \rho_{r, d}\right)
$$

where $\rho_{1,1}$ is constrained to enforce the trace-one normalisation. The Fisher information 
therefore, has the following block structure

$$
I(\rho)=\left(\begin{array}{ccc}
I^{d d}(\rho) & I^{d r}(\rho) & I^{d i}(\rho) \\
I^{r d}(\rho) & I^{r r}(\rho) & I^{r i}(\rho) \\
I^{i d}(\rho) & I^{i r}(\rho) & I^{i i}(\rho)
\end{array}\right)
$$

with the superscripts identifying the parameters considered; diagonal, real and imaginary. The weight matrix $G_{F}$ also has the same block structure with elements

$$
G_{F_{a, b}}=\operatorname{Tr}\left[\frac{\partial \rho_{\boldsymbol{\theta}}}{\partial \theta_{a}} \cdot \frac{\partial \rho_{\boldsymbol{\theta}}}{\partial \theta_{b}}\right]
$$

In the parametrisation described above, the weight matrix $G_{F}$ has the following block diagonal form:

(i) The diagonal-diagonal block:

(a) $G_{F_{a, b}}^{d d}=1+\delta_{a, b}$

(ii) The real-real and imaginary-imaginary block:

(a) $G_{F_{a, b}}^{r r / i i}=2 \cdot \delta_{a, b}$

with the other blocks being zero. We note that both the Fisher, and the weight matrix are of dimension $D:=2 r d-r^{2}-1$.

Lower bound on the smallest eigenvalue - As mentioned in the main text, we use Lemma 1 to bound the the smallest eigenvalue from below as

$$
G_{F}^{-1 / 2} \bar{I}\left(\rho_{0}\right) G_{F}^{-1 / 2} \leq G_{F}^{-1 / 2} \bar{I}(\rho) G_{F}^{-1 / 2} .
$$

Where $\rho_{0}$ is the state with $r$ equal eigenvalues and the same eigenvectors as the state $\rho$. The explicit form of $\bar{I}\left(\rho_{0}\right)$ is known, and has been evaluated in [31], and from it, we see that the minimum eigenvalue is lower bounded as $\mu_{\min } \geq r / r+1$ for $r>1$ and $\mu_{\min } \geq 1$ for pure states.

Upper bound on the largest eigenvalue-We use the inequality $I(\rho \mid \mathbf{s}) \leq F(\rho)$ between the classical and quantum Fisher informations to bound the largest eigenvalue of $G_{F}^{-1 / 2} I(\rho \mid \mathbf{s}) G_{F}^{-1 / 2}$ over all measurements by the largest eigenvalue of $G_{F}^{-1 / 2} F(\rho) G_{F}^{-1 / 2}$. The quantum Fisher information is calculated in the local parameterisation described above and evaluated at the state $\rho=\operatorname{Diag}\left(\lambda_{1}, \ldots, \lambda_{r}, \ldots, 0\right)$, diagonal in its eigenbasis. The details of this calculation can be found in [31], and we therefore avoid the repetition and merely state the elements of the matrix. Denoting $r_{a}, c_{a}$ to be the row and column positions of the element $a$ of the parameter vector $\boldsymbol{\theta}$, we have

(i) For the Diagonal-Diagonal block with $r>1$,

(a) $\left.F_{a, a}^{d d}\right|_{\boldsymbol{\theta}}=\frac{1}{\lambda_{r_{a}}}+\frac{1}{\lambda_{1}}$ when $r_{a} \leq r$

(b) $\left.F_{a, b}^{d d}\right|_{\boldsymbol{\theta}=\boldsymbol{\theta}_{0}}=\frac{1}{\lambda_{1}}$ when $r_{a}, r_{b} \leq r$, and $a \neq b$

(ii) For the Real-Real and Imaginary-Imaginary blocks:

(a) $\left.F_{a, a}^{r r / i i}\right|_{\boldsymbol{\theta}=\boldsymbol{\theta}_{0}}=\frac{4}{\lambda_{r_{a}}+\lambda_{c_{a}}}$ when $r_{a}<c_{a} \leq r$ 
(b) $\left.F_{a, a}^{r r / i i}\right|_{\boldsymbol{\theta}=\boldsymbol{\theta}_{0}}=\frac{4}{\lambda_{r_{a}}}$ when $r_{a} \leq r, c_{a}>r$

The off-diagonal blocks are zero. It is easy to see that the quantum Fisher matrix is upper bounded by the matrix $\frac{1}{\lambda_{\min }(\rho)} G_{F}^{d d} \bigoplus \frac{2}{\lambda_{\min }(\rho)} G_{F}^{r r} \bigoplus \frac{2}{\lambda_{\min }(\rho)} G_{F}^{i i}$. So we can write

$$
G_{F}^{-1 / 2} F G_{F}^{-1 / 2} \leq \frac{1}{\lambda_{\min }(\rho)} \mathbf{1}_{(r-1)} \bigoplus \frac{2}{\lambda_{\min }(\rho)} \mathbf{1}_{\left(2 r d-r^{2}+r\right)} \bigoplus \frac{2}{\lambda_{\min }(\rho)} \mathbf{1}_{\left(2 r d-r^{2}+r\right)}
$$

The maximum eigenvalue $\mu_{\max }$ is therefore upper bounded by $2 / \lambda_{\min }(\rho)$ for $r>1$, and 2 for $r=1$.

Combining the bounds to prove concentration-We can now substitute these values into the matrix Chernoff bound. While the value of the minimum/maximum eigenvalues differ for $r>1$ and $r=1$, we calculate the bound for the case when $r>1$, as this will provide a general bound for the number of settings required that holds even in the case of pure states. Writing $P_{\mathcal{S}}=G_{F}^{-1 / 2} I_{\mathcal{S}} G_{F}^{-1 / 2}$ and $\bar{P}=G_{F}^{-1 / 2} \bar{I} G_{F}^{-1 / 2}$ for notational simplicity, we have for $r>1$

$$
\mathbb{P}\left\{P_{\mathcal{S}} \notin[(1-\epsilon) \bar{P},(1+\epsilon) \bar{P}]\right\} \leq 2 D \cdot \exp \left(-k \frac{r \epsilon^{2} \lambda_{\min }(\rho)}{4(r+1) \cdot \log 2}\right):=\delta
$$

Therefore, with probability $1-\delta$ we have that

$$
(1-\epsilon) \bar{P} \leq P_{\mathcal{S}} \leq(1+\epsilon) \bar{P}
$$

This can be re-written in the form of inequalities of the MSE with $\epsilon>0$ sufficiently small

$$
(1-\epsilon) \operatorname{Tr}\left(\bar{P}^{-1}\right) \leq \operatorname{Tr}\left[P_{\mathcal{S}}^{-1}\right] \leq(1+\epsilon) \operatorname{Tr}\left(\bar{P}^{-1}\right)
$$

For a fixed value of $\epsilon$ and $\delta$, we see that the minimum number of settings $k$ required for the above abound to hold with probability greater than $1-\delta$ is

$$
k=\frac{C_{1}}{\lambda_{\min }(\rho)} \cdot \frac{(r+1)}{r} \log \left(\frac{2 D}{\delta}\right)
$$

where $C_{1}:=4\left(\log 2 / \epsilon^{2}\right)$ and $D:=2 r d-r^{2}-1$.

\section{References}

[1] Haroche S and Raimond J M 2013 Exploring the Quantum: Atoms, Cavities, and Photons (Oxford University Press)

[2] Wiseman H M and Milburn G J 2014 Quantum measurement and control (Cambridge University Press)

[3] Haffner H, Hansel W, Roos C F, Benhelm J, Chek-al kar D, Chwalla M, Korber T, Rapol U D, Riebe M, Schmidt P O, Becher C, Guhne O, Dur W and Blatt R 2005 Nature 643-646

[4] Gao W B, Lu C Y, Yao X C, Xu P, Guhne O, Goebel A, Chen Y A, Peng C Z, Chen Z B and Pan J W 2010 Nat Phys 331-335

[5] Banaszek K, D'Ariano G M, Paris M G A and Sacchi M F 1999 Physical Review A 61

[6] Blume-Kohout R 2010 Phys. Rev. Lett. 105200504

[7] James D F V, Kwiat P G, Munro W J and White A G 2001 Phys. Rev. A 64052312

[8] Řeháček J, Hradil Z c v, Knill E and Lvovsky A I 2007 Phys. Rev. A 75042108 
[9] Goncalves D S, Gomes-Ruggiero M A, Lavor C, Farias O J and Ribeiro P H S 2012 Journal of Quantum Information and Computation 12 775-790

[10] Vogel K and Risken H 1989 Phys. Rev. A 40(5) 2847-2849

[11] Bagan E, Ballester M A, Gill R D, Monras A and Muñoz Tapia R 2006 Phys. Rev. A 73032301

[12] Audenaert K M R and Scheel S 2009 New Journal of Physics 11023028

[13] Gonçalves D S, Lavor C, Gomes-Ruggiero M A, Cesário A T, Vianna R O and Maciel T O 2013 Phys. Rev. A 87052140

[14] Teo Y S, Englert B G, Řeháček J, Hradil Z and Mogilevtsev D 2012 New Journal of Physics 14105020

[15] Teo Y S, Zhu H, Englert B G, Řeháček J and Hradil Z c v 2011 Phys. Rev. Lett. 107020404

[16] Lvovsky A I and Raymer M G 2009 Rev. Mod. Phys. 81 299-332

[17] Monz T, Schindler P, Barreiro J T, Chwalla M, Nigg D, Coish W A, Harlander M, Hänsel W, Hennrich M and Blatt R 2011 Phys. Rev. Lett. 106130506

[18] Flammia S T, Gross D, Liu Y K and Eisert J 2012 New Journal of Physics 14095022

[19] Gross D, Liu Y K, Flammia S T, Becker S and Eisert J 2010 Phys. Rev. Lett. 105150401

[20] Carpentier A and KHKim A 2015 arXiv:1502.04654v2 [math.ST]

[21] Carpentier A, Eisert J, Gross D and Nickl R 2015 arXiv:1504.03234v1 [math.ST]

[22] Kalev A, Kosut R L and Deutsch I H 2015 arXiv:1502.00536v2

[23] Guţă M, Kypraios T and Dryden I 2012 New Journal of Physics 14105002

[24] Alquier P, Butucea C, Hebiri M, Meziani K and Morimae T 2013 Phys. Rev. A 88(3) 032113

[25] Butucea C, Guţă M and Kypraios T 2016 New Journal of Physics 18069501

[26] Cramer M, Plenio M B, Flammia S T, Somma R, Gross D, Bartlett S D, Landon-Cardinal O, Poulin D and Liu Y K 2010 Nat Commun 1149

[27] Catana C, Bouten L and Guță M 2015 Journal of Physics A: Mathematical and Theoretical 48365301

[28] Guță M and Kiukas J 2015 Communications in Mathematical Physics 335 1397-1428

[29] Kueng R, Rauhut H and Terstiege U 2015 Applied and Computational Harmonic Analysis - ISSN 1063-5203

[30] Kabanava M, Kueng R, Rauhut H and Terstiege U 2015 arXiv:1507.07184

[31] Acharya A, Kypraios T and Guţă M 2016 New Journal of Physics 18043018

[32] Candès E J, Strohmer T and Voroninski V 2013 Comm. Pure Appl. Math 66 1241-1274

[33] Gross D, Krahmer F and Kueng R 2015 Journal of Fourier Analysis and Applications 21 229-266

[34] Kimmel S and Liu Y K 2015 Quantum compressed sensing using 2-designs arXiv:1510.08887

[35] Kueng R 2015 Low rank matrix recovery from few orthonormal basis measurements 2015 Internation Conference on Sampling Theory and Applications (IEEE) pp 402-406

[36] Baldwin C H, Deutsch I H and Kalev A 2016 Phys. Rev. A 93(5) 052105

[37] Young G A and Smith R L 2005 Essentials of statistical inference (Cambridge University Press)

[38] Ahlswede R and Winter A 2002 IEEE Transactions on Information Theory 48 569-579

[39] Mahler D H, Rozema L A, Darabi A, Ferrie C, Blume-Kohout R and Steinberg A M 2013 Phys. Rev. Lett. 111(18) 183601

[40] Braunstein S L and Caves C M 1994 Phys. Rev. Lett. 72(22) 3439-3443

[41] Collins B and Śniady P 2006 Communications in Mathematical Physics 264 773-795

[42] Tao T 254 notes 1: Concentration of measure URL https: / /terrytao.wordpress . com/

[43] Bengtsson I and Życzkowski K 2008 Geometry of Quantum States: An Introduction to Quantum Entanglement 9780521891400 (Cambridge University Press)

[44] Koltchinskii V and Xia D 2015 Journal of Machine Learning Research 16 1757-1792

[45] Li N, Ferrie C, Gross J A, Kalev A and Caves C M 2016 Phy. Rev. Lett. 116180402

[46] Xia D and Koltchinskii V 2016 arXiv:1604.04600

[47] Qi B, Hou Z, Wang Y, Dong D, Zhong H S, Li L, Xiang G Y, Wiseman H M, Li C F and Guo G C 2015 arXiv:1512.01634

[48] Kravtsov K S, Straupe S S, Radchenko I V, Houlsby N M T, Huszár F and Kulik S P 2013 Phys. Rev. A 87(6) 062122

[49] Granade C, Ferrie C and Flammia S T 2016 arXiv:1605.05039

[50] Oszmaniec M, Augusiak R, Gogolin C, Kołodyński, Acin A and Lewenstein M 2016 Phys. Rev. X 6041044

[51] Rezakhani A T and Alipour S 2015 arXiv:1507.01736 [quant-ph] 